\title{
Multicenter Female Fabry Study (MFFS) - clinical survey on current treatment of females with Fabry disease
}

\author{
Malte Lenders ${ }^{1}$, Julia B. Hennermann², Christine Kurschat ${ }^{3}$, Arndt Rolfs ${ }^{4}$, Sima Canaan-Kühl ${ }^{5}$, Claudia Sommer ${ }^{6,7}$, \\ Nurcan Üçeyler ${ }^{6,7}$, Christoph Kampmann $^{2}$, Nesrin Karabul ${ }^{2,8}$, Anne-Katrin Giese $^{4}$, Thomas Duning ${ }^{9}$, \\ Jörg Stypmann ${ }^{10}$, Johannes Krämer ${ }^{7,11}$, Frank Weidemann ${ }^{7,12}$, Stefan-Martin Brand ${ }^{13}$, Christoph Wanner ${ }^{7}$ \\ and Eva Brand ${ }^{1 *}$
}

\begin{abstract}
Background: The aim of the present study was to assess manifestations of and applied treatment concepts for females with Fabry disease (FD) according to the current European Fabry Guidelines.

Methods: Between 10/2008 and 12/2014, data from the most recent visit of 261 adult female FD patients from six German Fabry centers were retrospectively analyzed. Clinical presentation and laboratory data, including plasma lyso-Gb3 levels were assessed.

Results: Fifty-five percent of females were on enzyme replacement therapy (ERT), according to recent European FD guidelines. Thirty-three percent of females were untreated although criteria for ERT initiation were fulfilled. In general, the presence of left ventricular hypertrophy $(\mathrm{LVH})$ seemed to impact more on ERT initiation than impaired renal function. In ERT-naïve females RAAS blockers were more often prescribed if LVH was present rather than albuminuria. Affected females with missense mutations showed a similar disease burden compared to females with nonsense mutations. Elevated plasma lyso-Gb3 levels in ERT-naïve females seem to be a marker of disease burden, since patients showed comparable incidences of organ manifestations even if they were $\sim 8$ years younger than females with normal lyso-Gb3 levels.
\end{abstract}

Conclusion: The treatment of the majority of females with FD in Germany is in line with the current European FD guidelines. However, a relevant number of females remain untreated despite organ involvement, necessitating a careful reevaluation of these females.

Keywords: Fabry disease, Females, Lyso-Gb3, Enzyme replacement therapy, Guidelines

\section{Background}

Fabry disease (FD; OMIM \#301500) is an X-linked (Xq22.1) inborn error of glycosphingolipid degradation resulting from deficient $\alpha$-galactosidase A activity (GLA; 300644) due to mutations in the GLA gene. Fabryspecific manifestations result from systemic lysosomal accumulation of mainly globotriaoslyceramide (Gb3) [1]. Gb3 accumulation in cells of different tissues is

\footnotetext{
* Correspondence: Eva.Brand@ukmuenster.de

${ }^{1}$ Internal Medicine D, Department of Nephrology, Hypertension and Rheumatology, University Hospital Muenster, Albert-Schweitzer-Campus 1, D-48149 Muenster, Germany

Full list of author information is available at the end of the article
}

accompanied by a high risk of early onset of stroke, lifethreatening arrhythmia, myocardial infarction, or cardiac and renal failure, leading to a reduced life expectancy [1]. Enzyme replacement therapy (ERT) with recombinant GLA, including agalsidase-alfa (Replagal, Shire) and agalsidase-beta (Fabrazyme, Genzyme) results in subcellular Gb3 clearance, leading to stabilization or at least slowing of disease progression in males and females [2-11]. While the onset of first symptoms (Fabry-associated pain, angiokeratoma, abdominal pain, cornea verticillata, hypo- or anhidrosis) in affected hemizygous males with low or absent enzymatic GLA activity starts in early childhood, heterozygous female 
patients may display much more variability in disease onset, severity, and progression. Because of this heterogeneous clinical picture in females, the optimal time point for ERT initiation still remains controversial.

Current FD guidelines and recommendations suggest ERT initiation in females with FD after the onset of first FD-typical renal, cardiac, and/or cerebral complications, or in rapidly progressive disease [12, 13]. According to Biegstraaten and colleagues, ERT should be considered in females with classical and non-classical phenotype if albuminuria/proteinuria, an estimated glomerular filtration rate (eGFR) $<90 \mathrm{ml} / \mathrm{min} / 1.73 \mathrm{~m}^{2}$, cardiac hypertrophy, signs of cardiac rhythm disturbances, cerebral white matter lesions, transient ischemic attack or stroke, FD-related pain or gastrointestinal (GI) symptoms are present [13].

However, since ERT is assumed to be most effective when started early before the onset of fibrosis or other irreversible tissue damage [14-16], this strategy might result in a therapeutic dilemma. Therefore, plasma lysoGb3 has been discussed as a prognostic marker for disease severity and progression. While this concept is established in males, the clinical relevance of lyso-Gb3 for female patients is still unclear [17-19].

In the Multicenter Female Fabry Study (MFFS), we retrospectively analyzed a cohort of 261 genetically confirmed adult female FD patients from six German Fabry centers to investigate the current ERT treatment status and the implementation of the latest European FD recommendations [13] concerning treatment strategies.

\section{Methods}

\section{Study design and patients}

Between 10/2008 and 12/2014, 261 genetically confirmed adult female FD patients were consecutively recruited or identified by family screenings (index patients were either affected males or females) at Fabry centers of the University Hospitals in Muenster, Wuerzburg, Mainz, Cologne, Rostock, and the Charité Berlin. Patients were retrospectively analyzed in an open cohort study. A comprehensive diagnostic work-up was performed in all centers including medical history and cardiac, renal, and neurological evaluation. Data documentation followed the clinical practice of the German Fabry Expert Centers for a rare multisystemic disorder. The detailed clinical work-up of patients has been reported elsewhere [20]. Gastrointestinal pain includes abdominal pain, tenesmus, or cramping more than once a week. Diarrhea was defined as $\geq 1$ day/ month with three loose bowels or $>250 \mathrm{~g}$ of stool weight per day. Fatigue was defined by the Fukuda criteria [21]. Cardiac assessment included echocardiography and electrocardiography. Left ventricular hypertrophy $(\mathrm{LVH})$ was defined as a septal diameter of $>12 \mathrm{~mm}$. Renal function was quantified by the eGFR using the Chronic Kidney
Disease-Epidemiology Collaboration equation (CKDEPI) [22] and the albumin-to-creatinine ratio (ACR) from spot urine. Renal impairment was defined as eGFR $<90 \mathrm{ml} / \mathrm{min} / 1.73 \mathrm{~m}^{2}$ according to "Kidney Disease: Improving Global Outcomes" (KDIGO) [23] and European FD [13] guidelines and albuminuria as ACR $>30 \mathrm{mg}$ albumin per $\mathrm{g}$ creatinine (microalbuminuria, $30-300 \mathrm{mg} / \mathrm{g}$; macroalbuminuria $>300 \mathrm{mg} / \mathrm{g}$ ). All patients underwent neurologic examination and a clinical interview focusing on a history of cerebral stroke or transient ischemic attack (TIA). Additionally, the presence of FD-related pain was investigated [24]. In a subset of patients with a characteristic history of acral pain and/or dysesthesias, small fiber neuropathy (SFN) was diagnosed additionally using quantitative sensory testing (QST) [25] and/or by determination of the intraepidermal nerve fiber density in skin biopsies [26, 27]. Disease severity was assessed using the Mainz Severity Score Index (MSSI) [28]. Additional medication was assessed for the current visit. Renin-angiotensin-aldosterone-system (RAAS) blockers include the prescription of angiotensin-converting enzyme blockers, angiotensin receptor blockers, renin blockers, as well as aldosterone antagonists. Diuretics include the prescription of high ceiling/loop diuretics, thiazides, carbonic anhydrase inhibitors, potassium-sparing diuretics. Analgesic drugs include the prescription of opioids, anticonvulsants, selective serotonin reuptake inhibitors, and non-steroidal anti-inflammatory drugs.

\section{GLA sequencing, measurement of GLA activity and plasma lyso-Gb3}

Genotyping for GLA gene mutations was performed by direct sequencing of all seven coding exons including adjacent intron-exons boundaries as previously reported [29].

The following described mutations/variations were detected: p.A20P, p.W24*, p.N34S, p.G35E, p.A37T, p.P40L, p.M42V, p.L45P, p.H46R, p.R49G, p.C53S, p.C63Y, p.I91T, p.C94S, p.R112C, p.R112H, p.R118C, p.A121T, p.S126G, p.S128G, p.L129P, p.L131P, p.A135V, p.N139S, p.A143T, p.G147R, p.Y151*, p.W162G, p.L167Q, p.C172Y, p.Y173*, p.G183S, p.M187R, p.M187V, p.W204*, p.K213M, p.N215S, p.R220*, p.N224S, p.R227Q, p.R227*, p.D231N, p.W236C, p.I242V, p.W262C, p.W262*, p.P265L, p.L268S, p.V269A, p.A288D, p.R301P, p.R301Q, p.R301*, p.D313Y, p.V316G, p.I319T, p.N320I, p.Q321*, p.Q327R, p.Q327E, p.G328R, p.E338X, p.W340*, p.R342Q, p.R342*, p.A350P, p.Q357*, p.W399*, IVS2 + 1G > T, IVS2 + 1G > A, IVS5 + 1G > A, IVS6 + 1G > A. An overview of newly detected GLA mutations is presented within the supplement (Additional file 1: Table S1). Females with genetic variants of unknown significance (GVUS) and polymorphisms (i.e. p.R112H; p.N139S; 
p.A143T; p.D313Y) were excluded $(n=37)$ from all calculations.

Nonsense mutations have been defined as single nucleotide exchanges, resulting in stop codons (termination), deletions or insertions of nucleotides resulting in a frame shift or large deletions within the protein, or splice site mutations, resulting in altered splice products of mRNA. GLA activity in leukocytes was determined using 4methylumbelliferyl- $\alpha$-D-galactopyranoside (Santa Cruz Biotechnology, Heidelberg, Germany) as previously described [30]. Nacetylgalactosamine (Santa Cruz Biotechnology) was used as specific inhibitor of endogenous $\alpha$ galactosidase B activity [31]. GLA enzyme activity was determined as nanomoles (nmol) of substrate hydrolyzed per hour (h) per mg protein and expressed as \% of reference. For plasma lyso-Gb3, lyso-ceramide was used as reference (Matreya LLC, Pleasant Gap, PA, USA) and D5-fluticasone propionate (EJY Tech, Inc., Rockville, Maryland, USA) served as internal standard. Plasma lyso-Gb3 levels (reference $0.9-2.3 \mathrm{ng} / \mathrm{ml}$ ) were measured at the University of Rostock (A. Rolfs), and at the University of Mainz (K. Lackner), Germany. To determine if patients were below or above these reference limits, each individual plasma lyso-Gb3 value was compared with the internal reference at the time-point of lyso-Gb3 determination.

\section{Statistical analysis}

Two-hundred sixty-one patients from the participating FD centers were included in the analysis. If not stated otherwise, continuous variables are expressed as mean with standard deviation or median with range. Categorical data is expressed as numbers and relative frequencies in percent. A quality control of assessed data is given in the supplement (Additional file 2: Table S2). An overall data completeness of $88.9 \%$ was achieved. To deal with missing data, analyses were performed with an as-is state for every parameter. Differences between groups were analyzed with the unpaired Student's $t$ or Mann-Whitney U test for continuous data, and the Fisher's exact test for categorical data. Statistical significance was considered at a 2 -sided $p<0.05$. All results are reported with their respective $95 \%$ confidence intervals (CI). SAS version 9.3 (SAS Institute Inc., Cary, North Carolina, USA) and GraphPad PRISM V5.0 software (GraphPad Software Inc., La Jolla, CA, USA) were used for all statistical analyses.

\section{Results \\ Clinical characterization of female patients with Fabry disease}

Between 10/2008 and 12/2014 a total of 261 genetically confirmed adult ( $\geq 18$ years) female FD patients presented at the participating Fabry centers in Muenster, Wuerzburg, Mainz, Cologne, Berlin, and Rostock. Since the presence of GVUS and polymorphisms might influence the outcome, females $(n=37)$ with these variants were excluded (i.e. p.R112H, $n=1$; p.N139S, $n=8$; p.A143T, $n=18$; p.D313Y, $n=10$ ). At the time of last visit and data analysis, 127 (56.7 \%) females received ERT (i.e. agalsidase-alfa $n=108$ [85.0\%], or agalsidasebeta $n=19$ [15.0\%]. The general ERT treatment status differed across centers from 46.2 to $77.8 \%$ females on ERT (Fig. 1).

Since the treatment period of patients overlapped with the worldwide agalsidase-beta shortage and a switch of product might have an influence on treatment outcome $[11,20]$, we assessed all changes in treatment regimens. During agalsidase-beta shortage, nearly all females (18 from 19) under agalsidase-beta received a dose-reduction $(0.5 \mathrm{mg} / \mathrm{kg}$ body weight) or switched to agalsidase-alfa until re-switch to full agalsidase-beta dose $(1.0 \mathrm{mg} / \mathrm{kg}$ body weight). From those who received agalsidase-alfa at data assessment $(n=108), 20$ patients had been switched from agalsidase-beta to -alfa. Consequently, in this "real world" study, both groups were not directly comparable. However, analysis of the clinical presentation revealed that patients under agalsidase-beta tended to be older $(p=0.1371)$ and showed a trend towards a longer ERT duration $(p=0.1499)$ than patients receiving agalsidasealfa (Additional file 3: Table S3). There were no significant differences between the two patient groups (Additional file 4: Table S4), except for angiokeratoma, NYHA classes, left ventricular diastolic diameter, resulting in a

female patients (\% of study)
under ERT (\% of center)
total included patients (\% under ERT)
Fig. 1 Overview of the participating FD centers and the distribution of female patients. Numbers and frequencies (\%) show either distributions
within the study (across all centers), or within each participating center


different left ventricular diastolic diameter index (Additional file 4: Table S4). To analyze whether patients were treated in accordance with current European FD guidelines [13] (class I: ERT is recommended; class IIA/B: ERT should/may be considered) at the onset of first organ manifestations, we analyzed the presence of at least one of these manifestations in different organs (cardiac: LVH, renal: eGFR $<90 \mathrm{ml} / \mathrm{min} / 1.73 \mathrm{~m}^{2}$, CNS: stroke/TIA, pain: FD-related neuropathic pain, gastrointestinal: gastrointestinal symptoms) justifying ERT indication according to classes I to IIA/B [13]. Following this stratification we classified the females into 4 groups: 1) females on ERT with indication $(n=124[55.4 \%]), 2)$ females without ERT with indication $(n=76[33.9 \%]), 3)$ females on ERT without indication $(n=3[1.3 \%]), 4)$ females without ERT without indication $(n=21$ [9.4 \%]) (Table 1, Fig. 2a). Since $34 \%$ of females were not on ERT, although an indication was identified, we further compared treated and untreated females with indications for ERT to identify differences between the two groups. The group of females on ERT (mean treatment duration: $68 \pm 48$ months) was older, and tended to comprise more patients with nonsense mutations $(p=0.0502)$, more patients with plasma lyso-Gb3 levels above the reference level, and more patients on additional medication (renin-angiotensin-aldosterone-system [RAAS] blockers, diuretics, analgesics) than females not receiving ERT (Table 1). Evaluation of clinical presentation further revealed that female patients on ERT showed a higher frequency of FD-typical signs and symptoms, such as angiokeratoma, edema (ankle), tinnitus, hypacusis, FD-related pain, and fatigue (Table 1). Patients on ERT suffered more often from stroke and demonstrated a higher MSSI score (Table 1). Evaluation of organ manifestations also showed that patients on ERT suffered more often from a cardiac involvement, resulting in higher frequencies of dyspnea and abnormalities in electrocardiogram (ECG) (defined as T-wave negativation). They presented with higher NYHA classes, had increased septal diameters, and higher frequencies for concentric LVH (RR: 1.6995 \% CI 1.37 to 2.08 ; $p<0.0001$; Table 2). Renal measures showed that patients on ERT also had a more severe renal impairment in that creatinine- and cystatin C-based eGFR values were severely decreased and showed a difference of $>15 \mathrm{ml} / \mathrm{min} / 1.73 \mathrm{~m}^{2}$ compared to non-treated female patients (Table 2). Due to the observed differences in eGFR, more patients on ERT were assigned to CKD stage $\geq 2$ than patients not on ERT (RR: $1.7095 \%$ CI 1.29 to 2.24; $p=0.0003$; Table 2). Interestingly, the general frequencies of patients with albuminuria were similar, although patients on ERT comprised more patients with macroalbuminuria (Table 2). Females on ERT presented with significantly more manifestations in different organs indicating ERT start [13] than untreated patients $(2.5[1-5]$ vs 1 [1-3], $p<0.0001$; Table 2, Fig. 2b).

To further investigate whether patients with nonsense mutations have a higher disease severity, we compared patients with missense and nonsense mutations separately from their ERT status (Table 3). All patients showed at least one manifestation justifying ERT treatment according to current guidelines [13]. Patients on ERT with nonsense mutations had a longer treatment duration than patients with missense mutations (Table 3). Furthermore, patients with nonsense mutations more often had elevated plasma lyso-Gb3 levels and angiokeratoma, and showed a trend towards more edema (ankle) ( $p=0.0541$; Table 3). However, no further significant differences in clinical presentation and cardiac as well as renal measures have been detected (Table 3). Also, patients showed no differences for additional medication with RAAS blockers, diuretics, or analgesics.

Untreated patients carrying nonsense mutations were slightly but not significantly younger than patients with missense mutations. Although untreated patients with nonsense mutations demonstrate more often a reduced GLA activity and tended to have higher plasma lyso-Gb3 levels $(p=0.0505)$, no further clinical differences in the group of patients without ERT have been detected (Table 3).

\section{Plasma lyso-Gb3 in ERT-naïve females with FD}

Since the biomarker lyso-Gb3 correlates well with disease manifestation and progression in male FD patients, we analyzed whether differences in plasma lyso-Gb3 levels might also explain differences in FD-specific manifestations and symptoms in female patients. Therefore, we compared ERT-naïve females (independent of a manifestation that would indicate ERT) with lyso-Gb3 levels below the reference value $(n=16)$ with those above the reference value $(n=52)$ (Table 4$)$. Females with lyso-Gb3 levels above the reference were younger than patients with normal lyso-Gb3 levels ( $41 \pm 13$ vs. $49 \pm 15$ years; $p=0.0299$ ). Females with elevated lysoGb3 levels comprised more patients with nonsense mutations (Table 4). Furthermore, females with elevated lyso-Gb3 levels suffered more often from FD-related pain (Table 4). Interestingly, evaluation of further FDtypical symptoms as well as cardiac and renal measures showed no intergroup differences. Also, the prescription of additional medication was similar in both groups. However, patients with elevated lyso-Gb3 levels tended to a higher albumin-creatinine-ratio (ACR; $p=0.0530$; Table 4).

\section{Discussion}

The aim of our multicenter study was to investigate the current ERT treatment status and the implementation of 
Table 1 Clinical presentation of female Fabry patients

\begin{tabular}{|c|c|c|c|c|c|}
\hline $\begin{array}{l}\text { Clinical presentation, laboratory } \\
\text { parameters and medication }\end{array}$ & $\begin{array}{l}\text { Females with ERT } \\
\text { with indication }\end{array}$ & $\begin{array}{l}\text { Females w/o ERT } \\
\text { with indication }\end{array}$ & $\begin{array}{l}\text { Females with ERT } \\
\text { without indication }\end{array}$ & $\begin{array}{l}\text { Females w/o ERT } \\
\text { without indication }\end{array}$ & Total \\
\hline $\mathrm{N}$ & $124(55.4)$ & $76(33.9)$ & $3(1.3)$ & $21(9.4)$ & 224 \\
\hline Age $[y]$ & $53 \pm 14^{* * * *}$ & $43 \pm 14$ & $44 \pm 9$ & $35 \pm 13$ & $48 \pm 15$ \\
\hline Weight [kg] & $71.0 \pm 17.1$ & $69.9 \pm 17.5$ & $66.2 \pm 11.2$ & $66.6 \pm 19.6$ & $70.1 \pm 17.4$ \\
\hline Height $[\mathrm{cm}]$ & $164.5 \pm 6.7$ & $165.8 \pm 13.8$ & $164.0 \pm 4.3$ & $166.7 \pm 6.2$ & $165.1 \pm 9.1$ \\
\hline Heart rate $[b p m]$ & $69 \pm 13$ & $69 \pm 11$ & $63 \pm 7$ & $67 \pm 8$ & $69 \pm 12$ \\
\hline $\mathrm{SBP}[\mathrm{mmHg}]$ & $122 \pm 17$ & $123 \pm 14$ & $126 \pm 5$ & $115 \pm 16$ & $122 \pm 16$ \\
\hline $\mathrm{DBP}[\mathrm{mmHg}]$ & $74 \pm 9$ & $77 \pm 9$ & $76 \pm 1$ & $73 \pm 10$ & $75 \pm 9$ \\
\hline Nonsense mutation [n] & $59(50.0)$ & $25(34.7)$ & $0(0.0)$ & $4(19.1)$ & $88(41.1)$ \\
\hline a-galactosidase A activity [\% reference] & $118 \pm 109$ & $118 \pm 95$ & $127 \pm 74$ & $135 \pm 72$ & $119 \pm 101$ \\
\hline a-galactosidase A activity below reference [n] & $42(56.0)$ & $24(53.3)$ & $1(33.3)$ & $3(42.9)$ & $70(53.9)$ \\
\hline Lyso-Gb3 value [ng/ml] & $7.5 \pm 6.5$ & $7.9 \pm 5.8$ & $4.5 \pm 0.3$ & $5.2 \pm 4.6$ & $7.4 \pm 6.1$ \\
\hline Lyso-Gb3 value above reference $[\mathrm{n}]$ & $70(94.6)^{* *}$ & $43(78.2)$ & $2(100.0)$ & $9(69.2)$ & $124(86.1)$ \\
\hline Pts on RAAS blockers [n] & $66(57.4)^{* * * *}$ & $16(23.9)$ & $0(0.0)$ & $3(18.8)$ & $85(42.3)$ \\
\hline Pts on diuretic drugs [n] & $45(39.1)^{* * * *}$ & $5(7.7)$ & $0(0.0)$ & $1(5.9)$ & $51(52.5)$ \\
\hline Pts on analgesic drugs [n] & $37(35.2)^{* * *}$ & $7(10.5)$ & $0(0.0)$ & $0(0.0)$ & $44(22.9)$ \\
\hline ERT since [months] & $68 \pm 48$ & $0 \pm 0$ & $43 \pm 34$ & $0 \pm 0$ & $38 \pm 49$ \\
\hline Angiokeratoma [n] & $59(49.6)^{* *}$ & $20(27.4)$ & $2(66.7)$ & $3(15.0)$ & $84(39.1)$ \\
\hline Edema [n] & $28(22.6)^{* * * *}$ & $1(1.4)$ & $0(0.0)$ & $0(0.0)$ & 29 (13.3) \\
\hline Gastrointestinal pain [n] & $32(26.2)$ & $26(36.6)$ & $0(0.0)$ & $0(0.0)$ & $58(27.1)$ \\
\hline Diarrhea [n] & $33(27.0)$ & $13(18.8)$ & $0(0.0)$ & $0(0.0)$ & $46(21.8)$ \\
\hline Hypohidrosis [n] & $49(40.5)$ & $22(29.3)$ & $2(66.7)$ & $1(5.0)$ & $74(33.8)$ \\
\hline Cornea verticillata [n] & $76(65.5)$ & $29(55.8)$ & $2(66.7)$ & $6(42.9)$ & $113(61.1)$ \\
\hline Tinnitus [n] & $42(34.2)^{*}$ & $13(18.1)$ & $1(33.3)$ & $2(9.5)$ & $58(26.5)$ \\
\hline Hypacusis [n] & $27(22.0)^{*}$ & $7(9.9)$ & $1(33.3)$ & $0(0.0)$ & $35(16.1)$ \\
\hline FD-related pain [n] & $82(66.1)^{*}$ & $37(49.3)$ & $0(0.0)$ & $0(0.0)$ & 119 (53.6) \\
\hline Neuropathic pain [n] & $40(32.8)^{* * *}$ & $7(9.7)$ & $0(0.0)$ & $0(0.0)$ & $47(21.7)$ \\
\hline Fatigue $[\mathrm{n}]$ & $62(51.2)^{* * * *}$ & $15(20.0)$ & $1(33.3)$ & $0(0.0)$ & $78(35.6)$ \\
\hline Ever stroke/TIA [n] & $29(23.4)^{*}$ & $7(9.2)$ & $0(0.0)$ & $0(0.0)$ & $36(16.1)$ \\
\hline SFN [n] & $22(29.3)$ & $9(15.0)$ & $0(0.0)$ & $2(12.5)$ & $33(21.7)$ \\
\hline
\end{tabular}

Disease severity score

MSSI score

$20.7 \pm 10.8^{* * * *}$

$7.3 \pm 5.2$

$7.3 \pm 5.0$

$1.5 \pm 1.5$

$14.7 \pm 11.4$

Categorical data are presented as $\mathrm{n}$ and are $\%$ of total in parenthesis. Otherwise data is presented as mean \pm standard deviation. eGFR: estimated glomerular filtration rate ( ${ }^{a}$ excluding patients with dialysis and/or kidney transplant). DBP: diastolic blood pressure; MSSI Mainz Severity Score Index, Pts patients, RAAS renin-angiotensin-aldosterone-system, SBP systolic blood pressure, SFN small fiber neuropathy, TIA transitory ischemic attack

$p<0.05,{ }^{* *} p<0.01,{ }^{* * *} p<0.001,{ }^{* * * *} p<0.0001$

the European FD guidelines and recommendations in female patients with FD in Germany.

Our main findings are: 1) Fifty-seven \% of female FD patients in Germany were under ERT, nearly all (except three females) presented with different organ manifestations that justify ERT initiation according to current European guidelines [13]; 2) one third of females remained without ERT, although indications (organ manifestations) for ERT initiation were fulfilled; 3) the presence of LVH seems to impact more on ERT initiation than impaired renal function; 4) in ERT-naïve females the prescription of RAAS blockers is mostly consistent with the presence of LVH, but not with albuminuria; 5) once affected females with missense mutations show similar disease burden compared to females with nonsense mutations; 6) elevated plasma lyso-Gb3 levels in ERT-naïve females seem to be a marker of disease burden, since these patients showed comparable incidences of organ manifestations and a higher frequency of FD-related pain even if they were $\sim 8$ years younger than females with normal lyso-Gb3 levels. 


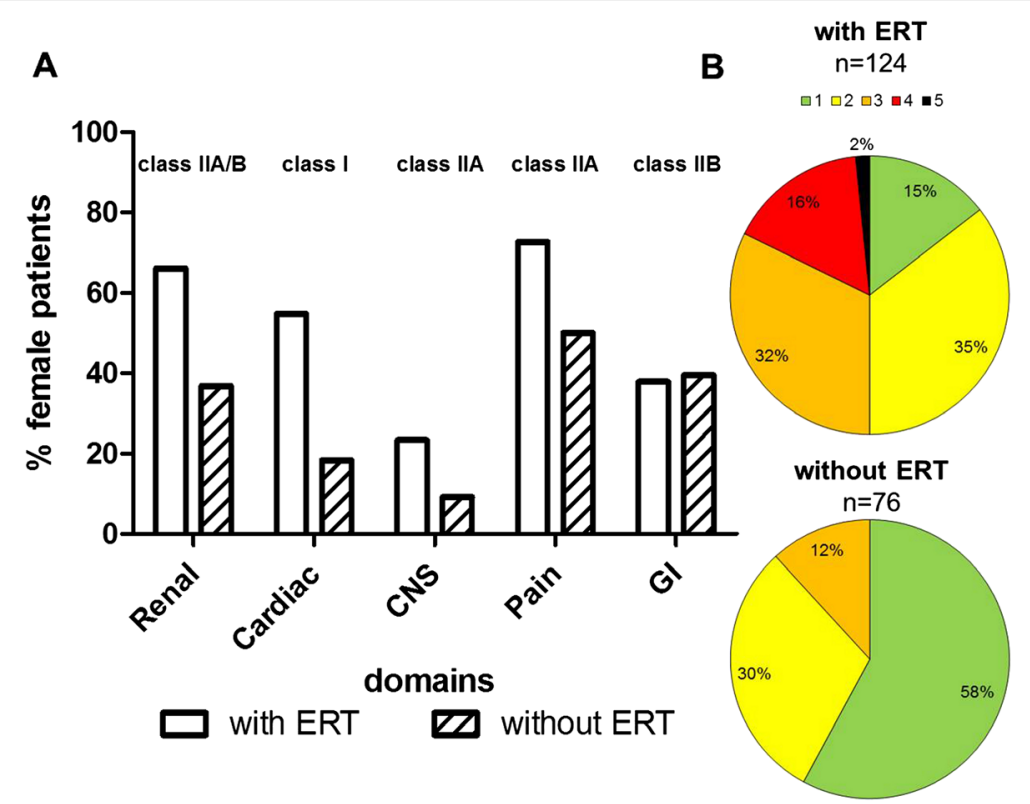

Fig. 2 Differences between patients with and without ERT at least one organ manifestation justifying ERT according to current guidelines. a Distribution of manifestations in different organs justifying ERT with class I (ERT is recommended) and class IIA/B (ERT should/may be considered) recommendations. b Accumulation of different organ manifestations justifying ERT. Females may present with one to five different manifestations (green, yellow, orange, red, black represent 1, 2, 3, 4, 5 simultaneous manifestations, respectively). Cardiac: presence of left ventricular hypertrophy; Renal: eGFR $<90 \mathrm{ml} / \mathrm{min} / 1.73 \mathrm{~m}^{2}$; CNS: stroke/ transient ischemic attack; Pain: neuropathic/FDrelated pain; Gl: gastrointestinal symptoms (diarrhea, abdominal pain)

The time point for ERT initiation in males with FD is clinically well established, since patients often suffer from a FD-typical organ involvement and/or display abnormal biomarkers (i.e. low or absent GLA activity, high plasma lyso-Gb3 values). In contrast, the optimal time point for ERT initiation in females with FD, often classified as "only" asymptomatic or minor symptomatic carriers still remains unclear, as their disease manifestations and progression, as well as biomarker levels are diverse.

Most guidelines recommend ERT initiation in females with FD as soon as FD-typical organ manifestations such as LVH, renal insufficiency, or clinical events such as TIA or stroke appear [12, 13]. Our analysis of 224 females with FD may reflect an average of women with FD in Germany. About $55 \%$ of the affected females are on ERT, confirming the prevalence of $53 \%$ reported in the Fabry Outcome Survey (FOS) registry (FOS Investigator report 2012-2013) and suggesting that the reported prevalence of $34 \%$ treated females within the Fabry Registry [32, 33] might be too low, at least for Germany.

Of those patients receiving ERT, nearly all showed at least one organ manifestation defined as LVH, an eGFR $<90 \mathrm{ml} / \mathrm{min} / 1.73 \mathrm{~m}^{2}$, cerebral complications, pain, or GI symptoms indicating ERT. Concerning this aspect, the treatment concept for females with FD in Germany is in line with the current European FD recommendations and guidelines [13]. Nevertheless, still one third of ERTnaïve female patients suffered from at least one clinically relevant organ manifestation. Even if the observed $\mathrm{LVH}$, renal insufficiency, or stroke and/or TIA might also be due to comorbidities and, more importantly, some patients refuse ERT, or can't receive ERT due to pregnancy or future family planning, the remaining number of clinically not justifiable untreated affected females seems to be relevant. As mentioned before, one reason for the relatively high frequency of ERT-untreated females with mild renal involvement only might be that many physicians did not recognize these females as patients at risk. In clinical practice, these patients may benefit from RAAS blockers. If under this treatment and with comorbidities/ risk factors (such as arterial hypertension) wellcontrolled, renal function still declines, ERT should be initiated. The high incidence and increased risk for LVH in patients on ERT indicates that the presence of $\mathrm{LVH}$ seems to impact more on ERT initiation than impaired renal function. These observations urge the need for a more stringent implementation of the new European FD guidelines for females across centers and reevaluation of untreated females. Furthermore, some female patients refusing ERT might benefit from future chaperone treatment strategies [34].

Renal biopsies demonstrate that even in children Gb3 accumulation within podocytes affects foot process effacement without presence of albuminuria, indicating an early Fabry nephropathy [35]. In fact, several studies suggest a better benefit when ERT is started at an early disease stage 
Table 2 Cardiac and renal parameters

\begin{tabular}{|c|c|c|c|c|c|}
\hline Cardiac measures & $\begin{array}{l}\text { Females with ERT } \\
\text { with indication }\end{array}$ & $\begin{array}{l}\text { Females w/o ERT } \\
\text { with indication }\end{array}$ & $\begin{array}{l}\text { Females with ERT } \\
\text { without indication }\end{array}$ & $\begin{array}{l}\text { Females w/o ERT } \\
\text { without indication }\end{array}$ & Total \\
\hline Dyspnea [n] & $54(43.6)^{* * *}$ & $14(18.7)$ & $0(0.0)$ & $1(4.8)$ & $69(30.9)$ \\
\hline \multicolumn{6}{|l|}{ NYHA class [n] } \\
\hline none & $29(28.2)$ & $31(45.6)^{*}$ & $1(50.0)$ & $9(56.3)$ & $70(37.0)$ \\
\hline । & $34(33.0)$ & $25(36.8)$ & $1(50.0)$ & $6(37.5)$ & $66(34.9)$ \\
\hline$\|$ & $27(26.2)$ & $11(16.2)$ & $0(0.0)$ & $1(6.3)$ & 39 (20.6) \\
\hline III & $13(12.6)^{* *}$ & $1(1.5)$ & $0(0.0)$ & $0(0.0)$ & $14(7.4)$ \\
\hline IV & $0(0.0)$ & $0(0.0)$ & $0(0.0)$ & $0(0.0)$ & $0(0.0)$ \\
\hline LV diastolic diameter [mm] & $45.8 \pm 5.5$ & $46.2 \pm 4.4$ & $42.5 \pm 3.5$ & $46.4 \pm 7.2$ & $46.0 \pm 5.3$ \\
\hline LV systolic diameter [mm] & $28.8 \pm 6.1$ & $28.5 \pm 4.7$ & $24.0 \pm 1.4$ & $29.7 \pm 4.9$ & $28.7 \pm 5.5$ \\
\hline LVDDi $\left[\mathrm{cm} / \mathrm{m}^{2}\right]$ & $2.79 \pm 0.33$ & $2.83 \pm 0.68$ & $2.58 \pm 0.12$ & $2.78 \pm 0.42$ & $2.81 \pm 0.49$ \\
\hline Septal diameter [mm] & $12.3 \pm 3.6^{* * * *}$ & $9.6 \pm 2.0$ & $9.5 \pm 2.1$ & $8.4 \pm 1.5$ & $11.0 \pm 3.3$ \\
\hline LVH [n] & $64(55.7)^{* * * *}$ & $14(18.9)$ & $0(0.0)$ & $0(0.0)$ & $78(37.0)$ \\
\hline Posterior wall diameter [mm] & $11.3 \pm 3.1^{* * * *}$ & $9.4 \pm 1.9$ & $10.5 \pm 0.7$ & $8.9 \pm 1.6$ & $10.4 \pm 2.8$ \\
\hline $\mathrm{RWT}[\mathrm{cm}]$ & $0.53 \pm 0.17^{* * * *}$ & $0.41 \pm 0.09$ & $0.48 \pm 0.11$ & $0.38 \pm 0.10$ & $0.47 \pm 0.15$ \\
\hline ECG abnormalities [n] & $42(36.5)^{* * * *}$ & $7(10.0)$ & $1(50.0)$ & $1(5.3)$ & $51(24.8)$ \\
\hline Pacemaker [n] & $18(14.5)^{* * *}$ & $0(0.0)$ & $0(0.0)$ & $0(0.0)$ & $18(8.0)$ \\
\hline Myocardial infarction [n] & $7(6.9)^{*}$ & $0(0.0)$ & $0(0.0)$ & $0(0.0)$ & $7(3.5)$ \\
\hline \multicolumn{6}{|l|}{ Renal measures } \\
\hline Albumin/creatinine-ratio $\left[\mathrm{mg} / \mathrm{g}_{\text {creatinine }}\right]$ & $48[0-4773]$ & $37[0-734]$ & $7[7]$ & 9 [0-226] & 39 [0-4773] \\
\hline Albuminuria [n] & $60(61.9)$ & $30(58.8)$ & $0(0.0)$ & $6(40.0)$ & $96(58.5)$ \\
\hline Microalbuminuria (30-300 mg/g creatinine) [n] & $40(66.7)^{*}$ & $27(90.0)$ & $0(0.0)$ & $6(100.0)$ & $73(76.0)$ \\
\hline Macroalbuminuria (>300 mg/g (reatinine) $[\mathrm{n}]$ & $20(33.3)$ & $3(10.0)$ & $0(0.0)$ & $0(0.0)$ & $23(24.0)$ \\
\hline Cystatin C [mg/l] & $1.03 \pm 0.36^{* * * *}$ & $0.78 \pm 0.19$ & $0.65 \pm 0.18$ & $0.71 \pm 0.09$ & $0.90 \pm 0.32$ \\
\hline $\mathrm{eGFR}_{\text {cys }}\left[\mathrm{ml} / \mathrm{min} / 1.73 \mathrm{~m}^{2}\right]^{\mathrm{a}}$ & $80.4 \pm 26.3^{* * * *}$ & $103.6 \pm 20.7$ & $116.1 \pm 20.3$ & $114.5 \pm 13.6$ & $92.6 \pm 26.7$ \\
\hline Creatinine $[\mathrm{mg} / \mathrm{dl}]$ & $0.99 \pm 0.93^{* * *}$ & $0.75 \pm 0.15$ & $0.64 \pm 0.11$ & $0.65 \pm 0.09$ & $0.87 \pm 0.71$ \\
\hline eGFR $R_{\text {creat }}\left[\mathrm{ml} / \mathrm{min} / 1.73 \mathrm{~m}^{2}\right]^{\mathrm{a}}$ & $81.2 \pm 25.3^{* * * *}$ & $98.6 \pm 19.1$ & $108.8 \pm 12.3$ & $113.8 \pm 12.6$ & $90.4 \pm 24.8$ \\
\hline $\mathrm{eGFR}_{\text {creat }}<90 \mathrm{ml} / \mathrm{min} / 1.73 \mathrm{~m}^{2}[\mathrm{n}]$ & $82(66.1)^{* * *}$ & $28(36.8)$ & $0(0.0)$ & $0(0.0)$ & $110(49.1)$ \\
\hline $\mathrm{Hb}[\mathrm{mg} / \mathrm{dl}]$ & $13.3 \pm 1.0$ & $13.4 \pm 0.99$ & $13.1 \pm 0.31$ & $13.3 \pm 1.1$ & $13.3 \pm 1.02$ \\
\hline Dialysis [n] & $2(1.6)$ & $0(0.0)$ & $0(0.0)$ & $0(0.0)$ & $2(0.9)$ \\
\hline Kidney transplantation [n] & $1(0.8)$ & $0(0.0)$ & $0(0.0)$ & $0(0.0)$ & $1(0.5)$ \\
\hline $\begin{array}{l}\text { Manifestations in different organs justifying ERT } \\
\text { (per patient) [n] }\end{array}$ & $2.5[1-5]^{* * * *}$ & $1[1-3]$ & - & - & $2[0-5]$ \\
\hline
\end{tabular}

Categorical data are presented as $\mathrm{n}$ and are $\%$ of total in parenthesis. Otherwise data is presented as mean \pm standard deviation or median [range]. eGFR: estimated glomerular filtration rate ('excluding patients with dialysis and/or kidney transplant); $H b$ Hemoglobin. eGFR $_{\text {creat }}$ and eGFR ${ }_{\text {cys }}$ are calculated via the CKDEPI formulas according to Levey et al. 2009 and Inker et al. 2012, respectively. LVDDi: left ventricular diastolic diameter index; LVH: left ventricular hypertrophy ( $>12 \mathrm{~mm}$ septal diameter); NYHA New York Heart Association, RWT relative wall thickness. ${ }^{*} p<0.05,{ }^{* *} p<0.01,{ }^{* * *} p<0.001,{ }^{* * * *} p<0.0001$

before irreversible organ damage such as fibrosis occurs $[9,14-16]$. Interestingly, in females, cardiac replacement fibrosis can already appear at a non-hypertrophic disease stage, being in contrast to males who generally develop LVH prior to replacement fibrosis [36, 37]. In our study, we did not include data on cardiac MRIs, since most of the participating centers routinely perform echocardiography but not cardiac MRI. Of note, in our subset of 68 cardiac MRIs, 41 females revealed late enhancement as a sign of cardiac fibrosis. Interestingly, 22 females with late enhancement did not yet present with LVH. Of these, 12 were not treated with ERT. Hence, in females with FD cardiac MRIs should be routinely performed to identify those with cardiac involvement, necessitating ERT initiation [37].

In addition, protective drugs such as RAAS blockers are needed in females (and males) with renal insufficiency, proteinuria, and LVH. In our study cohort, RAAS blockers were given in $>40 \%$ of all females with a $>2.0$-fold higher treatment frequency in 
Table 3 Clinical presentation of females with and without ERT with ERT indications with nonsense and missense mutations

\begin{tabular}{|c|c|c|c|c|}
\hline \multirow[b]{2}{*}{$\begin{array}{l}\text { Clinical presentation, laboratory } \\
\text { parameters and medication }\end{array}$} & \multicolumn{2}{|l|}{ Females with ERT } & \multicolumn{2}{|l|}{ Females without ERT } \\
\hline & $\begin{array}{l}\text { Mmissense mutations } \\
(n=65)\end{array}$ & $\begin{array}{l}\text { Nonsense mutations } \\
(n=59)\end{array}$ & $\begin{array}{l}\text { Missense mutations } \\
(n=51)\end{array}$ & $\begin{array}{l}\text { Nonsense mutations } \\
(n=25)\end{array}$ \\
\hline Age [y] & $53 \pm 14$ & $53 \pm 15$ & $45 \pm 15$ & $40 \pm 13$ \\
\hline ERT duration [months] & $57 \pm 47$ & $81 \pm 46^{* *}$ & - & - \\
\hline a-galactosidase A activity below reference [n] & $24(60.0)$ & $18(51.4)$ & $11(39.3)$ & $13(76.5)^{*}$ \\
\hline Lyso-Gb3 value $[\mathrm{ng} / \mathrm{ml}]$ & $6.3 \pm 4.1$ & $8.6 \pm 8.0$ & $6.9 \pm 6.0$ & $9.9 \pm 5.0$ \\
\hline Lyso-Gb3 value above reference [n] & $30(88.2)$ & $40(100.0)^{*}$ & $26(70.3)$ & $17(94.4)$ \\
\hline Angiokeratoma [n] & $25(39.7)$ & $34(60.7)^{*}$ & $10(20.4)$ & $10(41.7)$ \\
\hline Edema $[\mathrm{n}]$ & $10(15.4)$ & $18(30.5)$ & $0(0.0)$ & $1(4.2)$ \\
\hline Gastrointestinal pain [n] & $22(33.9)$ & $10(17.5)$ & $19(40.4)$ & $7(29.2)$ \\
\hline Hypohidrosis [n] & $22(34.4)$ & $27(47.4)$ & $15(29.4)$ & $7(29.7)$ \\
\hline Cornea verticillata [n] & $37(63.8)$ & $39(67.2)$ & $16(50.0)$ & $13(65.0)$ \\
\hline Tinnitus [n] & $23(35.9)$ & $19(32.2)$ & $9(18.8)$ & $4(16.7)$ \\
\hline Hypacusis [n] & 19 (24.6) & $11(19.0)$ & $4(8.3)$ & $3(13.0)$ \\
\hline FD-related pain [n] & $41(63.1)$ & $41(69.5)$ & $21(41.2)$ & $16(66.7)^{*}$ \\
\hline Fatigue $[\mathrm{n}]$ & $32(50.8)$ & $30(51.7)$ & $11(21.6)$ & $4(16.7)$ \\
\hline Ever TIA [n] & $4(6.4)$ & $9(15.8)$ & $2(4.1)$ & $2(8.3)$ \\
\hline Ever stroke $[\mathrm{n}]$ & $11(19.0)$ & $10(19.6)$ & $3(5.9)$ & $0(0.0)$ \\
\hline SFN [n] & $10(29.4)$ & $12(29.3)$ & $3(7.9)$ & $6(27.3)$ \\
\hline \multicolumn{5}{|l|}{ Disease severity score } \\
\hline MSSI score & $21.8 \pm 12.0$ & $19.6 \pm 9.4$ & $7.5 \pm 5.3$ & $6.9 \pm 5.1$ \\
\hline \multicolumn{5}{|l|}{ Cardiac measures } \\
\hline Septal diameter $[\mathrm{mm}]$ & $11.8 \pm 2.7$ & $12.8 \pm 4.4$ & $9.6 \pm 2.2$ & $9.6 \pm 1.7$ \\
\hline LVH [n] & $33(55.0)$ & $31(56.4)$ & $10(20.0)$ & $4(16.7)$ \\
\hline Posterior wall diameter [mm] & $11.2 \pm 2.8$ & $11.5 \pm 3.4$ & $9.5 \pm 1.9$ & $9.1 \pm 1.7$ \\
\hline $\mathrm{RWT}[\mathrm{cm}]$ & $0.52 \pm 0.14$ & $0.54 \pm 0.21$ & $0.42 \pm 0.10$ & $0.39 \pm 0.07$ \\
\hline Pacemaker [n] & $10(15.4)$ & $8(13.6)$ & $0(0.0)$ & $0(0.0)$ \\
\hline Myocardial infarction [n] & $3(5.7)$ & $4(8.3)$ & $0(0.0)$ & $0(0.0)$ \\
\hline \multicolumn{5}{|l|}{ Renal measures } \\
\hline Albumin/creatinine-ratio $\left[\mathrm{mg} / \mathrm{g}_{\text {Creatinine }}\right]$ & $66[0-1148]$ & $39[0-4773]$ & $37[0-734]$ & 36 [6-535] \\
\hline Albuminuria [n] & $34(64.2)$ & $26(59.1)$ & $19(55.9)$ & $11(64.7)$ \\
\hline Creatinine $[\mathrm{mg} / \mathrm{dl}]$ & $1.07 \pm 1.24$ & $0.89 \pm 0.34$ & $0.76 \pm 0.16$ & $0.74 \pm 0.12$ \\
\hline eGFR $R_{\text {creat }}\left[\mathrm{ml} / \mathrm{min} / 1.73 \mathrm{~m}^{2}\right]^{a}$ & $81.3 \pm 25.4$ & $81.0 \pm 25.3$ & $96.7 \pm 19.8$ & $101.0 \pm 18.4$ \\
\hline $\begin{array}{l}\text { Manifestations in different organs justifying ERT } \\
\text { (per patient) [n] }\end{array}$ & $2[1-5]$ & $3[1-4]$ & $1[1-3]$ & $1[1-3]$ \\
\hline
\end{tabular}

Nonsense mutations include single nucleotide exchanges, resulting in a stop codon (termination), deletion or insertions of nucleotides resulting in a frame shift or large deletions within the protein, or splice site mutations, resulting in altered splice products of mRNA. Categorical data are presented as $\mathrm{n}$ and are $\%$ of total in parenthesis. Otherwise data are presented as mean \pm SD or median [range]. eGFR estimated glomerular filtration rate ( ${ }^{\mathrm{a}}$ excluding patients with dialysis and/or kidney transplant), LVH Left ventricular hypertrophy, MSSI Mainz Severity Score Index, SFN small fiber neuropathy, RWT relative wall thickness, TIA transitory

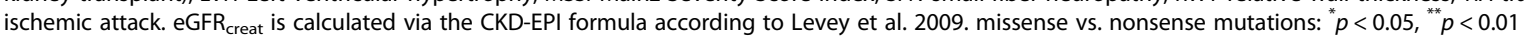

females on ERT compared to those without ERT (57\% vs. $24 \%)$. In general, the frequency of renoprotective and cardioprotective RAAS blocker prescription was comparable across FD centers and in accordance with the frequencies of LVH and albuminuria only in females on ERT. In patients without ERT, a similar relation was found for the presence of LVH and RAAS blockers, but not with albuminuria. Since albuminuria/proteinuria is one of the first signs of impaired renal function, and according to recent KDIGO guidelines [23], persistent albuminuria is associated with poor clinical outcome a more aggressive renoprotective intervention should be considered in these patients. 
Table 4 Differences in clinical presentation between ERT-naïve females below and above the plasma lyso-Gb3 reference values

\begin{tabular}{|c|c|c|}
\hline $\begin{array}{l}\text { Clinical presentation, } \\
\text { laboratory parameters } \\
\text { and medication }\end{array}$ & $\begin{array}{l}\text { Females below the } \\
\text { reference }(n=16)\end{array}$ & $\begin{array}{l}\text { Females above the } \\
\text { reference }(n=52)\end{array}$ \\
\hline Age [y] & $49 \pm 15$ & $41 \pm 13^{*}$ \\
\hline Nonsense mutation $[\mathrm{n}]$ & $1(6.3)$ & $19(36.5)^{*}$ \\
\hline Lyso-Gb3 value [ng/ml] & $0.9 \pm 0.5$ & $9.4 \pm 5.0^{* *}$ \\
\hline FD-related pain [n] & $2(12.5)$ & $25(48.1)^{*}$ \\
\hline Ever TIA/ stroke [n] & $3(18.8)$ & $4(8.0)$ \\
\hline SFN [n] & $1(6.3)$ & $8(16.7)$ \\
\hline \multicolumn{3}{|l|}{ Disease severity score } \\
\hline MSSI Score & $4.3 \pm 3.8$ & $6.8 \pm 5.9$ \\
\hline \multicolumn{3}{|l|}{ Cardiac measures } \\
\hline Septal diameter [mm] & $9.1 \pm 1.4$ & $9.4 \pm 2.1$ \\
\hline LVH [n] & $2(12.5)$ & $7(14.0)$ \\
\hline $\begin{array}{l}\text { Posterior wall } \\
\text { diameter [mm] }\end{array}$ & $9.2 \pm 1.8$ & $9.4 \pm 1.9$ \\
\hline $\mathrm{RWT}[\mathrm{cm}]$ & $0.41 \pm 0.09$ & $0.40 \pm 0.09$ \\
\hline Pacemaker [n] & $0(0.0)$ & $0(0.0)$ \\
\hline \multicolumn{3}{|l|}{ Renal measures } \\
\hline $\begin{array}{l}\text { Albumin/creatinine-ratio } \\
{\left[\mathrm{mg} / \mathrm{g}_{\text {creatinine }}\right]}\end{array}$ & $9[5-51]$ & $36[0-734]$ \\
\hline Albuminuria [n] & $2(28.6)$ & $21(56.8)$ \\
\hline Creatinine [mg/dl] & $0.76 \pm 0.21$ & $0.74 \pm 0.13$ \\
\hline $\mathrm{eGFR}_{\text {creat }}\left[\mathrm{ml} / \mathrm{min} / 1.73 \mathrm{~m}^{2}\right]^{\mathrm{a}}$ & $96.1 \pm 26.2$ & $100.7 \pm 18.9$ \\
\hline $\begin{array}{l}\text { Manifestations in different } \\
\text { organs justifying ERT } \\
\text { (per patient) [n] }\end{array}$ & $1[0-3]$ & $1[0-3]$ \\
\hline
\end{tabular}

Categorical data are presented as $\mathrm{n}$ and are \% of total in parenthesis. Otherwise data is presented as mean \pm SD or median [range]. eGFR estimated glomerular filtration rate, $L V H$ left ventricular hypertrophy, MSSI Mainz Severity Score Index, RWT relative wall thickness, SFN small fiber neuropathy, TIA Transitory ischemic attack. ${ }^{*} p<0.05,{ }^{* *} p<0.0001$

Interestingly, at the time-point of data assessment, we observed no clinically relevant differences concerning FD-manifestations and disease burden between females receiving either agalsidase-alfa or agalsidase-beta. Although the relative small group of agalsidase-betareceiving patients might be a limitation, it seems that physicians do not prefer any of these two drugs to treat more severe affected female patients.

Since ERT initiation in female patients based on the clinical presentation might be too late, a prognostic biomarker identifying females with a rapid disease progression is warranted. While plasma lyso-Gb3 seems to be a useful prognostic marker to elucidate disease severity and progression in affected males and correlates well with ERT effectiveness [17, 38, 39], lyso-Gb3 levels are difficult to correlate with disease burden and progression in females and patients with non-classical GLA mutations $[17-19,39]$. In our study, plasma lyso-Gb3 levels above the reference were more frequent in females on ERT compared to treatment-naïve females. ERT-naïve female patients with elevated lyso-Gb3 levels showed comparable incidences of organ manifestations and a higher frequency of FD-related pain, even if they were 8 years younger than females with normal lyso-Gb3 levels. Therefore, our preliminary data suggest that elevated plasma lyso-Gb3 levels in females also seem to be a marker of disease burden. However, further longitudinal observational studies are warranted to clarify the prognostic value of lyso-Gb3 in ERT-naïve females with different lyso-Gb3 levels.

In contrast to most males, females with FD present with a heterogeneous clinical picture and variable disease progression, independent of the presence of a nonsense or a missense mutation. Our results demonstrate that although females with missense mutations seem to have a lower risk to suffer from severe FD-typical manifestations and events justifying ERT in general, the disease burden in once affected patients is similar, independent of the disease-causing underlying GLA mutation (i.e. nonsense or missense). The variation and the missing genotype-phenotype correlation might be due to the controversially discussed X-chromosomal inactivation. While Elstein and colleagues did not find any associations with clinical signs in 77 females FD patients analyzed for skewed X-inactivation [40], Echevarria and colleagues demonstrated significant differences in disease progression depending on the direction and degree of X-inactivation in 56 patients [41]. In that respect, also larger longitudinal studies are warranted to confirm these results and to demonstrate an effect of skewed Xchromosomal inactivation on FD phenotype in affected females.

\section{Conclusions}

We conclude that the treatment concept for females with FD in Germany is in line with the current European Fabry guidelines. However, a relevant number of females remain untreated despite organ involvement, necessitating a careful reevaluation of these females. Elevated plasma lyso-Gb3 levels in females seem to be a marker of disease burden, even if the prognostic value of elevated lyso-Gb3 levels for ERT initiation in females needs evaluation in future observational studies.

\section{Limitations}

The intention of this study was to present a current clinical overview of females with FD and their treatment status and to assess the implementation of current European FD guidelines [13] for ERT treatment. Analysis and readout of techniques such as echoes have been performed within each center by the same on-side specialized operators, which limits intra-center variations. 
However, slight variations between participating centers cannot be fully excluded. In addition, some parameters such as plasma lyso-Gb3 or GLA activity were not available for the entire study cohort. Since no longitudinal analysis starting with treatment-naïve baselines has been performed and treatment regimens of agalsidase-alfa and -beta groups were heterogeneous, no comparisons of effectiveness between agalsidase-alfa or-beta could be drawn.

\section{Additional files}

Additional file 1: Table S1. Summary of newly identified coding GLA mutations and all identified deletions/insertions. (DOC $53 \mathrm{~kb}$ )

Additional file 2: Table S2. Quality control of assessed data for analyzed patients $(n=224)$. (DOC $33 \mathrm{~kb})$

Additional file 3: Table S3. Differences in clinical presentation between females receiving agalsidase-alfa and -beta. (DOC $40 \mathrm{~kb}$ )

Additional file 4: Table S4. Differences in cardiac and renal measures between females receiving agalsidase-alfa and -beta. (DOC $38 \mathrm{~kb}$ )

\section{Abbreviations}

$A C R$, albumin/creatinine ratio; $\mathrm{Cl}$, confidence interval; $C K D$, chronic kidney disease; CNS, central nervous system; ECG, electrocardiogram; eGFR, estimated glomerular filtration rate; ERT, enzyme replacement therapy; FD, Fabry disease; Gb3, globotriaosylceramide; GLA, alpha-galactosidase A; GVUS, genetic variance of unknown significance; KDIGO, kidney disease improving global outcomes; LVH, left ventricular hypertrophy; MFFS, Multicenter Female Fabry Study; MSSI, Mainz Severity Score Index; NYHA, New York Heart Association; $\mathrm{PCR}$, polymerase chain reaction; $\mathrm{QST}$, quantitative sensory testing; RAAS, renin-angiotensin-aldosterone-system; RR, relative risk; SFN, small fiber neuropathy; TIA, transient ischemic attack.

\section{Acknowledgements}

We thank Anne Huster, Samira Schiwek, Irina Turkin, and Jutta Beilker for expert technical assistance. This study was funded by Shire.

\section{Funding}

The funding company had no role in the design and conduct of the study, in the collection, analysis, and interpretation of the data, or in the preparation, review, or approval of the manuscript. The researchers were independent of the funding company.

\section{Availability of data and materials}

All available data supporting the conclusions of this article is published in the manuscript. Individual patient records have not been included due to patients' privacy.

\section{Authors' contributions}

All authors have contributed to the article by participating in the conception and design (ML, SMB, EB), acquisition of data (ML, JS, SR, FW, JK, CKu, CKa, JK, AKG, SC, EB) or analysis and interpretation of data (ML, JBH, CK, AR, SCK, FW, $\mathrm{CW}, \mathrm{SMB}, \mathrm{EB})$, drafting the article $(\mathrm{ML}, \mathrm{SMB}, \mathrm{EB})$ or revising it critically for important intellectual content (FW, CW, CKu, CKa, SCK, NK, CS, NÜ, JS, TD, AKG ). All authors read and approved the final version of the manuscript.

\section{Competing interests}

$\mathrm{CKu}, \mathrm{FW}, \mathrm{NK}, \mathrm{SCK}, \mathrm{AR}$, and $\mathrm{EB}$ have received speaker honoraria from Genzyme, Shire and Amicus. Research grants were given to the institutions (Wuerzburg and Muenster) by Genzyme and Shire. SMB has received speaker honoraria from Shire. CW is a member of the Fabry Registry board of advisors and has received speaker honoraria from Genzyme. ML has received speaker honoraria and travel grants from Shire and Genzyme. JK has received speaker honoraria and travel grants from Genzyme. NÜ has received travel grants, speaker honoraria, and research grants from Genzyme and travel grants from Shire. CS has received speaker honoraria and research support from Genzyme. CKa have received travel support and speaker honoraria from Shire, Genzyme, and Amicus and research grants from Shire. JBH has received speaker honoraria from Shire, Genzyme, and Amicus, travel support from Shire and Genzyme, and research grants from Shire.

Ethics approval and consent to participate

All investigations were performed after approval of the Ethical Committees of the participating centers (Medical Association of Westfalian-Lippe and the Ethical Committee of the Medical Faculty of the University of Muenster; Ethical Committee of the Medical Faculty of the University of Cologne; Ethical Committee of the Medical Faculty of the University of Wuerzburg; Ethical Committees of the Charité Berlin, University of Rostock, and University of Mainz; project-no: 2011-347-f; 14-328) and written informed consent for molecular analysis and publication was obtained from all patients.

\section{Author details}

${ }^{1}$ Internal Medicine D, Department of Nephrology, Hypertension and Rheumatology, University Hospital Muenster, Albert-Schweitzer-Campus 1, D-48149 Muenster, Germany. ${ }^{2}$ Villa Metabolica, Department for Pediatric and Adolescent Medicine, University Medical Center of the Johannes Gutenberg-University Mainz, Mainz, Germany. ${ }^{3}$ Department II of Internal Medicine and center for Molecular Medicine Cologne, University of Cologne, Cologne, Germany. ${ }^{4}$ Albrecht-Kossel-Institute for Neuroregeneration, University of Rostock, Rostock, Germany. ${ }^{5}$ Department of Medicine, Division of Nephrology, University Hospital Charité, Campus Virchow-Klinikum, Berlin, Germany. ${ }^{6}$ Department of Neurology, University of Wuerzburg, Wuerzburg, Germany. ${ }^{7}$ Department of Medicine, Divisions of Cardiology and Nephrology, Comprehensive Heart Failure Center, Fabry Center for Interdisciplinary Therapy (FAZIT), University of Wuerzburg, Wuerzburg, Germany. ${ }^{8}$ Department of Neuropediatrics and Inborn Metabolic Disorders, Centre for Rare Diseases, University Children's Hospital, St. Josefs-Hospital, Bochum, Germany.

${ }^{9}$ Department of Neurology, University Hospital Muenster, Muenster, Germany. ${ }^{10}$ Department of Cardiovascular Medicine, Division of Cardiology, University Hospital Muenster, Muenster, Germany. ${ }^{11}$ Department of Pediatrics and Adolescence Medicine, University of Ulm, Ulm, Germany.

${ }^{12}$ Katharinen-Hospital Unna, Unna, Germany. ${ }^{13}$ Institute of Sports Medicine, Molecular Genetics of Cardiovascular Disease, University Hospital Muenster, Muenster, Germany.

Received: 4 April 2016 Accepted: 17 June 2016

Published online: 29 June 2016

\section{References}

1. Zarate YA, Hopkin RJ. Fabry's disease. Lancet. 2008;372:1427-35.

2. Eng CM, Guffon N, Wilcox WR, Germain DP, Lee P, Waldek S, Caplan L, Linthorst GE, Desnick RJ. Safety and efficacy of recombinant human alphagalactosidase A-replacement therapy in Fabry's disease. N Engl J Med. 2001:345:9-16.

3. Schiffmann R, Kopp JB, Austin 3rd HA, Sabnis S, Moore DF, Weibel T, Balow JE, Brady RO. Enzyme replacement therapy in Fabry disease: A randomized controlled trial. JAMA. 2001;285:2743-9.

4. Banikazemi M, Bultas J, Waldek S, Wilcox WR, Whitley CB, McDonald M, Finkel R, Packman S, Bichet DG, Warnock DG, Desnick RJ; Fabry Disease Clinical Trial Study Group. Agalsidase-beta therapy for advanced Fabry disease: A randomized trial. Ann Intern Med. 2007;146:77-86.

5. Hughes DA, Elliott PM, Shah J, Zuckerman J, Coghlan G, Brookes J, Mehta $A B$. Effects of enzyme replacement therapy on the cardiomyopathy of Anderson-Fabry disease: A randomised, double-blind, placebo- controlled clinical trial of agalsidase-alfa. Heart. 2008;94:153-8.

6. Wraith JE, Tylki-Szymanska A, Guffon N, Lien YH, Tsimaratos M, Vellodi A, Germain DP. Safety and efficacy of enzyme replacement therapy with agalsidase beta: An international, open-label study in pediatric patients with Fabry disease. J Pediatr. 2008;152:563-70.

7. Mehta A, Beck M, Elliott P, Giugliani R, Linhart A, Sunder-Plassmann G, Schiffmann R, Barbey F, Ries M, Clarke JT; Fabry Outcome Survey investigators. Enzyme replacement therapy with agalsidase alfa in patients with Fabry's disease: An analysis of registry data. Lancet. 2009;374:1986-96.

8. Whybra C, Miebach E, Mengel E, Gal A, Baron K, Beck M, Kampmann C. A 4-year study of the efficacy and tolerability of enzyme replacement therapy with agalsidase alfa in 36 women with Fabry disease. Genet Med. 2009;11:441-9. 
9. Weidemann F, Niemann M, Störk S, Breunig F, Beer M, Sommer C, Herrmann S, Ertl G, Wanner C. Long-term outcome of enzyme-replacement therapy in advanced Fabry disease: evidence for disease progression towards serious complications. J Intern Med. 2013;274:331-41.

10. Lenders M, Karabul N, Duning T, Schmitz B, Schelleckes M, Mesters R, Hense HW, Beck M, Brand SM, Brand E. Thromboembolic events in Fabry disease and the impact of factor V Leiden. Neurology. 2015;84:1009-16.

11. Lenders $M$, Canaan-Kühl S, Krämer J, Duning T, Reiermann S, Sommer C, Stypmann J, Blaschke D, Üçeyler N, Hense HW, Brand SM, Wanner C, Weidemann F, Brand E. Patients with Fabry disease after enzyme replacement therapy dose reduction and switch-2-year follow-up. J Am Soc Nephrol. 2016;27:952-62.

12. Eng CM, Germain DP, Banikazemi M, Warnock DG, Wanner C, Hopkin RJ, Bultas J, Lee P, Sims K, Brodie SE, Pastores GM, Strotmann JM, Wilcox WR. Fabry disease: guidelines for the evaluation and management of multiorgan system involvement. Genet Med. 2006;8:539-48.

13. Biegstraaten M, Arngrímsson R, Barbey F, Boks L, Cecchi F, Deegan PB, Feldt- Rasmussen U, Geberhiwot T, Germain DP, Hendriksz C, Hughes DA, Kantola I, Karabul N, Lavery C, Linthorst GE, Mehta A, van de Mheen E, Oliveira JP, Parini R, Ramaswami U, Rudnicki M, Serra A, Sommer C, SunderPlassmann G, Svarstad E, Sweeb A, Terryn W, Tylki-Szymanska A, Tøndel C, Vujkovac B, Weidemann F, Wijburg FA, Woolfson P, Hollak CE. Recommendations for initiation and cessation of enzyme replacement therapy in patients with Fabry disease: the European Fabry Working Group consensus document. Orphanet J Rare Dis. 2015;10:36.

14. Germain DP, Waldek S, Banikazemi M, Bushinsky DA, Charrow J, Desnick RJ, Lee P, Loew T, Vedder AC, Abichandani R, Wilcox WR, Guffon N. Sustained, long-term renal stabilization after 54 months of agalsidase beta therapy in patients with Fabry disease. J Am Soc Nephrol. 2007;18:1547-57.

15. Weidemann F, Niemann M, Breunig F, Herrmann S, Beer M, Störk S, oelker W, Ertl G, Wanner C, Strotmann J. Long-term effects of enzyme replacement therapy on Fabry cardiomyopathy: evidence for a better outcome with early treatment. Circulation. 2009;119:524-9.

16. Feriozzi S, Torras J, Cybulla M, Nicholls K, Sunder-Plassmann G, West M, FOS Investigators. The effectiveness of long-term agalsidase alfa therapy in the treatment of Fabry nephropathy. Clin J Am Soc Nephrol. 2012;7:60-9.

17. Germain DP. Fabry disease. Orphanet J Rare Dis. 2010;5:30.

18. Wang RY, Bodamer OA, Watson MS, Wilcox WR, ACMG Work Group on Diagnostic Confirmation of Lysosomal Storage Diseases. Lysosomal storage diseases: diagnostic confirmation and management of presymptomatic individuals. Genet Med. 2011;13:457-84.

19. Laney DA, Bennett RL, Clarke V, Fox A, Hopkin RJ, Johnson J, O'Rourke E, Sims K, Walter G. Fabry disease practice guidelines: Recommendations of the national society of genetic counselor. J Genet Counsel. 2013;22:555-64.

20. Weidemann F, Krämer J, Duning T, Lenders M, Canaan-Kühl S, Krebs A, Guerrero González H, Sommer C, Üçeyler N, Niemann M, Störk S, Schelleckes M, Reiermann S, Stypmann J, Brand SM, Wanner C, Brand E. Patients with Fabry disease after enzyme replacement therapy dose reduction versus treatment switch. J Am Soc Nephrol. 2014:25:837-49.

21. Fukuda K, Straus SE, Hickie I, Sharpe MC, Dobbins JG, Komaroff A, International Chronic Fatigue Syndrome Study Group. The chronic fatigue syndrome: A comprehensive approach to its definition and study. Ann Intern Med. 1994;121:953-9.

22. Levey AS, Stevens LA, Schmid CH, Zhang YL, Castro 3rd AF, Feldman HI, Kusek JW, Eggers P, Van Lente F, Greene T, Coresh J; CKD-EPI (Chronic Kidney Disease Epidemiology Collaboration). A new equation to estimate glomerular filtration rate. Ann Intern Med. 2009;150:604-12.

23. Kidney Disease: Improving Global Outcomes (KDIGO) CKD Work Group: KDIGO. Clinical Practice Guideline for the Evaluation and Management of Chronic Kidney Disease. Kidney inter Suppl. 2012;2013(3):1-150.

24. Üçeyler N, Ganendiran S, Kramer D, Sommer C. Characterization of pain in Fabry disease. Clin J Pain. 2014;30:915-20.

25. Rolke R, Baron R, Maier C, Tölle TR, Treede RD, Beyer A, Binder A, Birbaumer N, Birklein F, Bötefür IC, Braune S, Flor H, Huge V, Klug R, Landwehrmeyer GB, Magerl W, Maihöfner C, Rolko C, Schaub C, Scherens A, Sprenger T, Valet M, Wasserka B. Quantitative sensory testing in the German Research Network on Neuropathic Pain (DFNS): standardized protocol and reference values. Pain. 2006;123:231-43.

26. Devigili G, Tugnoli V, Penza P, Camozzi F, Lombardi R, Melli G, Broglio L, Granieri E, Lauria G. The diagnostic criteria for small fibre neuropathy: from symptoms to neuropathology. Brain. 2008;131:1912-25.
27. Lauria G, Hsieh ST, Johansson O, Kennedy WR, Leger JM, Mellgren SI, Nolano M, Merkies IS, Polydefkis M, Smith AG, Sommer C, Valls-Solé J; European Federation of Neurological Societies; Peripheral Nerve Society. European Federation of Neurological Societies/Peripheral Nerve Society Guideline on the use of skin biopsy in the diagnosis of small fiber neuropathy. Report of a joint task force of the European Federation of Neurological Societies and the Peripheral Nerve Society. Eur J Neurol. 2010;17:903-12.

28. Whybra C, Kampmann C, Krummenauer F, Ries M, Mengel E, Miebach E, Baehner F, Kim K, Bajbouj M, Schwarting A, Gal A, Beck M. The Mainz Severity Score Index: a new instrument for quantifying the Anderson-Fabry disease phenotype, and the response of patients to enzyme replacement therapy. Clin Genet. 2004;65:299-307.

29. Rolfs A, Martus P, Heuschmann PU, Grittner U, Holzhausen M, Tatlisumak T, Böttcher T, Fazekas F, Enzinger C, Ropele S, Schmidt R, Riess O, Norrving Bi sifap1 Investigators. Protocol and methodology of the stroke in young Fabry patients (sifap1) study: A prospective multicenter European study of 5,024 young stroke patients aged 18-55 years. Cerebrovasc Dis. 2011:31:253-62.

30. Desnick RJ, Allen KY, Desnick SJ, Raman MK, Bernlohr RW, Krivit W. Fabry's disease: enzymatic diagnosis of hemizygotes and heterozygotes. Alphagalactosidase activities in plasma, serum, urine, and leukocytes. J Lab Clin Med. 1973;81:157-71.

31. Mayes JS, Scheerer JB, Sifers RN, Donaldson ML. Differential assay for lysosomal alpha-galactosidases in human tissues and its application to Fabry's disease. Clin Chim Acta. 1981;112:247-51.

32. Wilcox WR, Oliveira JP, Hopkin RJ, Ortiz A, Banikazemi M, Feldt-Rasmussen U, Sims K, Waldek S, Pastores GM, Lee P, Eng CM, Marodi L, Stanford KE, Breunig F, Wanner C, Warnock DG, Lemay RM, Germain DP; Fabry Registry. Females with Fabry disease frequently have major organ involvement: lessons from the Fabry Registry. Mol Genet Metab. 2008;93:112-28.

33. Mehta A, Beck M, Eyskens F, Feliciani C, Kantola I, Ramaswami U, Rolfs A, Rivera A, Waldek S, Germain DP. Fabry disease: a review of current management strategies. QJM. 2010;103:641-59.

34. Giugliani R, Waldek S, Germain DP, Nicholls K, Bichet DG, Simosky JK, Bragat AC, Castelli JP, Benjamin ER, Boudes PF. A Phase 2 study of migalastat hydrochloride in females with Fabry disease: Selection of population, safety and pharmacodynamic effects. Mol Genet Metab. 2013;109:86-92.

35. Tøndel C, Kanai T, Larsen KK, Ito S, Politei JM, Warnock DG, Svarstad E. Foot process effacement is an early marker of nephropathy in young classic Fabry patients without albuminuria. Nephron. 2015;129:16-21.

36. Niemann M, Herrmann S, Hu K, Breunig F, Strotmann J, Beer M, Machann W, Voelker W, Ertl G, Wanner C, Weidemann F. Differences in fabry cardiomyopathy between female and male patients: consequences for diagnostic assessment. JACC Cardiovasc Imaging. 2011;4:592-601.

37. Weidemann F, Sanchez-Niño MD, Politei J, Oliveira JP, Wanner C, Warnock DG, Ortiz A. Fibrosis: a key feature of Fabry disease with potential therapeutic implications. Orphanet J Rare Dis. 2013:8:116.

38. Rombach SM, Dekker N, Bouwman MG, Linthorst GE, Zwinderman AH, Wijburg FA, Kuiper S, Vd Bergh Weerman MA, Groener JE, Poorthuis BJ, Hollak CE, Aerts JM. Plasma globotriaosylsphingosine: diagnostic value and relation to clinical manifestations of Fabry disease. Biochim Biophys Acta. 2010;1802:741-8.

39. Smid BE, van der Tol L, Biegstraaten M, Linthorst GE, Hollak CE, Porthuis BJ. Plasma globotriaosylsphingosine in relation to phenotypes of Fabry disease. J Med Genet. 2015;52:262-8.

40. Elstein D, Schachamorov E, Beeri R, Altarescu G. X-inactivation in Fabry disease. Gene. 2012;505:266-8.

41. Echevarria L, Benistan K, Toussaint A, Dubourg O, Hagege AA, Eladari D, Jabbour F, Beldjord C, De Mazancourt P, Germain DP. X-chromosome inactivation in female patients with Fabry disease. Clin Genet. 2016;89:44-54. 